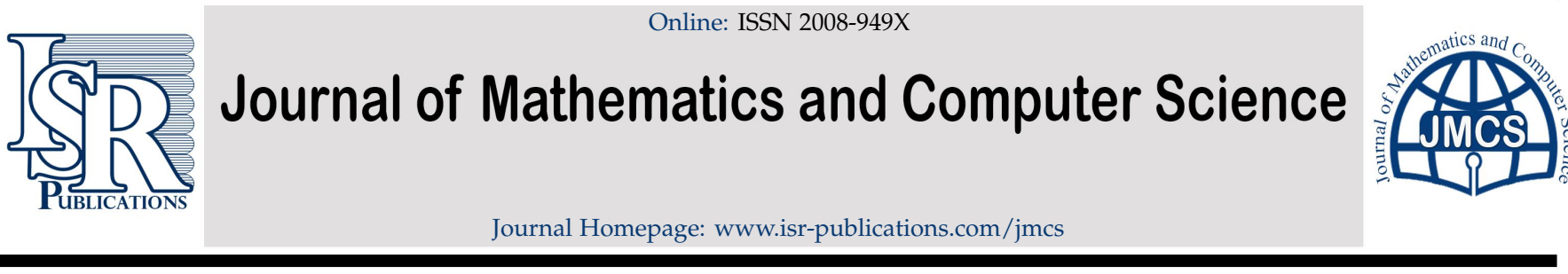

\title{
The Jensen's inequality and functional form of Jensen's in- equality for 3-convex functions at a point
}

\author{
Yu Ming Chuª, Imran Abbas Baloch ${ }^{\mathrm{b}, \mathrm{c}, *}$, Absar UI Haq ${ }^{\mathrm{d}}$, Manuel De La Sen ${ }^{\mathrm{e}}$ \\ ${ }^{a}$ Department of Mathematics, Huzhou University, Huzhou, China. \\ ${ }^{b}$ Abdus Salam School of Mathematical Sciences GC University, Lahore, Pakistan. \\ ${ }^{c}$ Higher Education Department, Govt. College Gulberg Lahore, Punjab, Pakistan. \\ ${ }^{d}$ Department of Natural Sciences and Humanities, University of Engineering and Technology (Narowal Campus), Lahore 54000, \\ Pakistan. \\ ${ }^{e}$ Institute of Research and Development of Processors, University of the Basque Country campus of Leioa (Bizkaia), 48940 Leioa, \\ Spain.
}

\begin{abstract}
In this paper, we give the refinement of extension of Jensen's inequality to affine combinations. Furthermore, we present a functional form of Jensen's inequality for continuous 3-convex functions at a point of one variable.
\end{abstract}

Keywords: Affine combination, positive linear functional, convex function, 3-convex functions at a point, Jensen's inequality. 2020 MSC: 26D15, 26A51, 26D10, 26A15.

(C)2021 All rights reserved.

\section{Introduction}

A subset $A$ of a linear space $X$ is called affine if it contains all binomial affine combination $p a_{1}+q a_{2}$, where $a_{1}, a_{2} \in A$ and $p, q \in \mathbb{R}$ such that $p+q=1$. The affine hull of a set $A \subseteq X$, which is denoted by aff $A$ is the smallest affine set that contains $A$. A function $f: A \rightarrow \mathbb{R}$ is called affine function, if the following equation holds for all binomial affine combinations of $A$

$$
f\left(p a_{1}+q a_{2}\right)=p f\left(a_{1}\right)+q f\left(a_{2}\right) .
$$

A subset $C$ of linear space $X$ is called convex if it contains all binomial convex combinations $\mathrm{pc}_{1}+\mathrm{qc}_{2}$ of points $c_{1}, c_{2} \in C$, where $p, q \in \mathbb{R}_{+}$and $p+q=1$. The convex hull of $C$ is the smallest convex set that contains $\mathrm{C}$ and denoted as convC. A function $\mathrm{f}: \mathrm{C} \rightarrow \mathbb{R}$ is convex if the inequality

$$
f\left(p c_{1}+q c_{2}\right) \leqslant p f\left(c_{1}\right)+q f\left(c_{2}\right)
$$

\footnotetext{
${ }^{*}$ Corresponding author

Email address: iabbasbaloch@gmail.com (Imran Abbas Baloch)

doi: $10.22436 /$ jmcs.022.02.05
}

Received: 2020-04-07 Revised: 2020-06-09 Accepted: 2020-06-22 
holds for all binomial convex combinations $p c_{1}+q c_{2}$ of points $c_{1}, c_{2} \in C$.

Let $\mathbb{X}$ be a subspace of linear space of all real functions on a nonempty set $\Omega$ and contains unit function $\mathrm{I}(\mathrm{x})=1$ for every $x \in \Omega$. For an interval $\mathcal{J} \subseteq \mathbb{R}$, the subset $\mathbb{X}_{\mathcal{J}} \subseteq \mathbb{X}$ contains all functions with image in J. If $p f_{1}+q f_{2}$ is a convex combination of functions $f_{1}, f_{2} \in \mathbb{X}_{\mathcal{J}}$, then the number (convex combination) $p f_{1}(x)+q f_{2}(x)$ is in $\mathcal{J}$ for every $x \in \Omega$, which indicates that functions set $\mathbb{X}_{\mathcal{J}}$ is convex. A linear functional $L: \mathbb{X} \rightarrow \mathbb{R}$ is positive (non-negative) if $L(f)>0(L(f) \geqslant 0)$ for every non-negative function $f \in \mathbb{X}$, and unital (normalized) if $L(1)=1$. In 2015, Pavić [4] gave the extension of Jensen's inequality to affine combinations in the following form.

Theorem 1.1. Let $\mathrm{p}_{\zeta}, \mathrm{q}_{\eta}, \mathrm{r}_{\xi} \geqslant 0$ be coefficients such that their sum $\mathrm{p}=\sum_{\zeta=1}^{\mathrm{n}} \mathrm{p}_{\zeta}, \mathrm{q}=\sum_{\eta=1}^{\mathrm{m}} \mathrm{q}_{\eta}, \mathrm{r}=\sum_{\xi=1}^{\mathrm{l}} \mathrm{r}_{\xi}$ satisfy $\mathrm{p}+\mathrm{q}-\mathrm{r}=1$ for $\mathrm{p}, \mathrm{q} \in(0,1]$ and $\mathrm{a}_{\zeta}, \mathrm{b}_{\eta}, \mathrm{c}_{\xi} \in \mathbb{R}$ be points such that $\mathrm{c}_{\xi} \in \operatorname{conv}\{\mathrm{a}, \mathrm{b}\}$, where

$$
a=\frac{1}{p} \sum_{\zeta=1}^{n} p_{\zeta} a_{\zeta}, b=\frac{1}{q} \sum_{\eta=1}^{m} a_{\eta} b_{\eta}
$$

Then the affine combination

$$
\sum_{\zeta=1}^{n} p_{\zeta} a_{\zeta}+\sum_{\eta=1}^{m} q_{\eta} b_{\eta}-\sum_{\xi=1}^{l} r_{\xi} c_{\xi}
$$

belongs to $\operatorname{conv}\{a, b\}$, and for every convex functions $f: \operatorname{conv}\left\{a_{\zeta}, b_{\eta}\right\} \rightarrow \mathbb{R}$ satisfies the inequality

$$
f\left(\sum_{\zeta=1}^{n} p_{\zeta} a_{\zeta}+\sum_{\eta=1}^{m} q_{\eta} b_{\eta}-\sum_{\xi=1}^{l} r_{\xi} c_{\xi}\right) \leqslant \sum_{\zeta=1}^{n} p_{\zeta} f\left(a_{\zeta}\right)+\sum_{\eta=1}^{m} q_{\eta} f\left(b_{\eta}\right)-\sum_{\xi=1}^{l} r_{\xi} f\left(c_{\xi}\right) .
$$

In 2014, Pavić [3] gave the functional form of jensen's inequality for the continuous convex functions of one variable in the following form.

Theorem 1.2. Let $[a, b] \subseteq \mathcal{J}$, for closed interval $\mathcal{J}$ and functions $g \in \mathbb{X}_{[a, b]}, h \in \mathbb{X}_{\mathcal{J} \backslash(a, b)}$ are defined, respectively. Furthermore, let $\mathrm{f}: \mathcal{J} \rightarrow \mathbb{R}$ be a continuous convex function such that $\mathrm{f}(\mathrm{g}), \mathrm{f}(\mathrm{h}) \in \mathbb{X}$. If a pair of unital positive linear functionals $\mathrm{L}, \mathrm{H}: \mathbb{X} \rightarrow \mathbb{R}$ satisfies

$$
\mathrm{L}(\mathrm{g})=\mathrm{H}(\mathrm{h}),
$$

then

$$
L(f(g)) \leqslant H(f(h)) .
$$

Furthermore, Pavić [3] also gave some consequent results based on the following corollaries:

Corollary 1.3. Let $\mathrm{f}: \mathcal{J} \rightarrow \mathbb{R}$ be a continuous convex function such that $\mathrm{f}(\mathrm{g}) \in \mathbb{X}$. If a unital positive linear functional $\mathrm{L}: \mathbb{X} \rightarrow \mathbb{R}$ satisfies (1.2) and (1.3) for $\mathrm{L}=\mathrm{H}$, then

$$
f(L(g)) \leqslant L(f(g)) .
$$

Corollary 1.4. Consider a sequence of closed intervals $\left[a_{1}, b_{1}\right] \subseteq \cdots \subseteq\left[a_{n-1}, b_{n-1}\right] \subseteq \mathcal{J}$, function $g_{1} \in \mathbb{X}_{\left[a_{1}, b_{1}\right]}$,

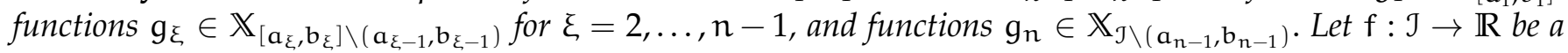
continuous convex function such that $\mathrm{f}\left(\mathrm{g}_{\zeta}\right) \in \mathbb{X}$. If an $\mathrm{n}$-tuple of unital positive linear functionals $\mathrm{L}_{\zeta}: \mathbb{X} \rightarrow \mathbb{R}$ satisfies

$$
\mathrm{L}_{\zeta}\left(\mathrm{g}_{\zeta}\right)=\mathrm{L}_{\zeta+1}\left(\mathrm{~g}_{\zeta+1}\right) \text { for } \zeta=1, \ldots, \mathrm{n}-1,
$$

then

$$
\mathrm{L}_{\zeta}\left(\mathrm{f}\left(\mathrm{g}_{\zeta}\right)\right) \leqslant \mathrm{L}_{\zeta+1}\left(\mathrm{f}\left(\mathrm{g}_{\zeta+1}\right)\right) \text { for } \zeta=1, \ldots, \mathrm{n}-1 \text {. }
$$


Corollary 1.5. Consider a closed interval $\mathcal{J} \subseteq \mathbb{R}$ and $\mathrm{g}_{1}, \ldots, \mathrm{g}_{\mathrm{n}} \in \mathbb{X}_{\mathcal{J}}$. If $\mathrm{f}: \mathcal{J} \rightarrow \mathbb{R}$ be a continuous convex function such that $\mathrm{f}\left(\mathrm{g}_{\zeta}\right) \in \mathbb{X}$, then every $\mathrm{n}$-tuple of positive linear functionals $\mathrm{L}_{\zeta}: \mathbb{X} \rightarrow \mathbb{R}$ with $\sum_{\zeta=1}^{n} \mathrm{~L}_{\zeta}(1)=1$ satisfies the inclusion

$$
\sum_{\zeta=1}^{n} L_{\zeta}\left(g_{\zeta}\right) \in \mathcal{J}
$$

and the inequality

$$
f\left(\sum_{\zeta=1}^{n} L_{\zeta}\left(g_{\zeta}\right)\right) \leqslant \sum_{\zeta=1}^{n} L_{\zeta}\left(f\left(g_{\zeta}\right)\right)
$$

Theorem 1.6. Consider a closed interval $\mathcal{J} \subseteq \mathbb{R},[a, b] \subseteq \mathcal{J}, g_{1}, \ldots, g_{n} \in \mathbb{X}_{[a, b]}, h_{1}, \ldots, h_{m} \in \mathbb{X}_{\mathcal{J} \backslash(a, b)}$. Let $\mathrm{f}: \mathcal{J} \rightarrow \mathbb{R}$ be a continuous convex function such that $\mathrm{f}\left(\mathrm{g}_{\zeta}\right), \mathrm{f}\left(\mathrm{h}_{\eta}\right) \in \mathbb{X}$. If a pair of $n$-tuple of positive linear functionals $\mathrm{L}_{\zeta}, \mathrm{H}_{\eta}: \mathbb{X} \rightarrow \mathbb{R}$ with $\sum_{\zeta=1}^{n} \mathrm{~L}_{\zeta}(1)=\sum_{\eta=1}^{m} \mathrm{H}_{\eta}(1)=1$ satisfies

$$
\sum_{\zeta=1}^{n} L_{\zeta}\left(g_{\zeta}\right)=\sum_{\eta=1}^{m} H_{\eta}\left(h_{\eta}\right)
$$

then

$$
\sum_{\zeta=1}^{n} L_{\zeta}\left(f\left(g_{\zeta}\right)\right) \leqslant \sum_{\eta=1}^{m} H_{\eta}\left(f\left(h_{\eta}\right)\right)
$$

In [1], Baloch et al defined a new class of functions which is defined as follow.

Definition 1.7. Let $c \in \mathcal{J}^{\circ}$, where $\mathcal{J}$ is an arbitrary interval (open, closed or semi-open in either direction) in $\mathbb{R}$ and $\mathcal{J}^{\circ}$ is its interior. We say that $f: \mathcal{J} \rightarrow \mathbb{R}$ is 3-convex function at point $c$ (respectively 3-concave function at point $c$ ) if there exists a constant $A$ such that the function $F(x)=f(x)-\frac{A}{2} x^{2}$ is concave (resp. convex) on $\mathcal{J} \cap(-\infty, c]$ and convex (resp. concave) on $\mathcal{J} \cap[c, \infty)$.

The function is 3-convex on an interval if and only if it is 3-convex at every point of the interval (see [1]) and the class of 3-convex functions at point $c$ is denoted as $K_{c}^{1}(\mathcal{J})\left(K_{c}^{2}(\mathcal{J})\right.$ ) (respectively 3-concave functions at point $\mathrm{c}$ ).

\section{Main results}

Theorem 2.1. Let $\mathrm{p}_{\zeta}, \mathrm{q}_{\eta}, \mathrm{r}_{\xi} \geqslant 0$ and $\lambda_{\zeta,}, \mu_{\eta}, v_{\xi} \geqslant 0$ be coefficients such that their sum $\mathrm{p}=\sum_{\zeta=1}^{n} \mathrm{p}_{\zeta}, \mathrm{q}=$ $\sum_{\eta=1}^{m} q_{\eta}, r=\sum_{\xi=1}^{l} r_{\xi}$ satisfy $p+q-r=1$ and $p, q \in(0,1] ; \lambda=\sum_{\zeta=1}^{n} \lambda_{\zeta}, \mu=\sum_{\eta=1}^{m} \mu_{\eta}, v=\sum_{\xi=1}^{l} v_{\xi}$ satisfy $\lambda+\mu-v=1$ and $\lambda, \mu \in(0,1]$. Let $a_{\zeta}, b_{\eta}, c_{\xi} \in I \subseteq \mathbb{R}$ be points such that $c_{\xi} \in \operatorname{conv}\{a, b\}$ and also $r_{\zeta}, s_{\eta}, t_{\xi} \in I \subseteq \mathbb{R}$ be points such that $t_{\xi} \in \operatorname{conv}\{r, s\}$, where

$$
a=\frac{1}{p} \sum_{\zeta=1}^{n} p_{\zeta} a_{\zeta}, b=\frac{1}{q} \sum_{\eta=1}^{m} q_{\eta} b_{\eta}, r=\frac{1}{p} \sum_{\zeta=1}^{n} \lambda_{\zeta} r_{\zeta}, s=\frac{1}{\mu} \sum_{\eta=1}^{m} \mu_{\eta} s_{\eta},
$$

if

$$
\begin{aligned}
& \sum_{\zeta=1}^{n} p_{\zeta}\left(a_{\zeta}\right)^{2}+\sum_{\eta=1}^{m} q_{\eta}\left(b_{\eta}\right)^{2}-\sum_{\xi=1}^{l} r_{\xi}\left(c_{\xi}\right)^{2}\left(\sum_{\zeta=1}^{n} p_{\zeta} a_{\zeta}+\sum_{\eta=1}^{m} q_{\eta} b_{\eta}-\sum_{\xi=1}^{l} r_{\xi} c_{\xi}\right)^{2} \\
& =\sum_{\zeta=1}^{n} \lambda_{\zeta}\left(r_{\zeta}\right)^{2}+\sum_{\eta=1}^{m} \mu_{\eta}\left(s_{\eta}\right)^{2}-\sum_{\xi=1}^{l} v_{\xi}\left(t_{\xi}\right)^{2}-\left(\sum_{\zeta=1}^{n} \lambda_{\zeta} r_{\zeta}+\sum_{\eta=1}^{m} \mu_{\eta} s_{\eta}-\sum_{\xi=1}^{l} v_{\xi} t_{\xi}\right)^{2}
\end{aligned}
$$


and also there exists $\mathrm{c} \in \mathcal{J}^{\circ}$ such that

$$
\max \left\{\max _{\zeta}\left\{a_{\zeta}\right\}, \max _{\eta}\left\{b_{\eta}\right\}, \max _{\xi}\left\{c_{\xi}\right\}\right\} \leqslant c \leqslant \min \left\{\min _{\zeta}\left\{r_{\zeta}\right\}, \min _{\eta}\left\{s_{\eta}\right\}, \min _{\xi}\left\{t_{\xi}\right\}\right\},
$$

then for every $\mathrm{f} \in \mathrm{K}_{\mathrm{c}}^{1}(\mathcal{J})$, the following inequality holds

$$
\begin{aligned}
& \sum_{\zeta=1}^{n} p_{\zeta} f\left(a_{\zeta}\right)+\sum_{\eta=1}^{m} q_{\eta} f\left(b_{\eta}\right)-\sum_{\xi=1}^{l} r_{\xi} f\left(c_{\xi}\right)-f\left(\sum_{\zeta=1}^{n} p_{\zeta} a_{\zeta}+\sum_{\eta=1}^{m} q_{\eta} b_{\eta}-\sum_{\xi=1}^{l} r_{\xi} c_{\xi}\right) \\
& \leqslant \sum_{\zeta=1}^{n} p_{\zeta} f\left(r_{\zeta}\right)+\sum_{\eta=1}^{m} q_{\eta} f\left(s_{\eta}\right)-\sum_{\xi=1}^{l} r_{\xi} f\left(t_{\xi}\right)-f\left(\sum_{\zeta=1}^{n} p_{\zeta} r_{\zeta}+\sum_{\eta=1}^{m} q_{\eta} s_{\eta}-\sum_{\xi=1}^{l} r_{\xi} t_{\xi}\right) .
\end{aligned}
$$

Proof. Since $f \in K_{c}^{1}(\mathcal{J})$, then there exists a constant $A$ such that $F(x)=f(x)-\frac{A}{2} x^{2}$ is concave on $I \cap(-\infty, c]$ and for $a_{\zeta}, b_{\eta}, c_{\xi} \in I \cap(-\infty, c]$ be points such that $c_{\xi} \in \operatorname{conv}\{a, b\}$, so by using inequality (1.1) we have

$$
\begin{aligned}
0 \geqslant & \sum_{\zeta=1}^{n} p_{\zeta} F\left(a_{\zeta}\right)+\sum_{\eta=1}^{m} q_{\eta} F\left(b_{\eta}\right)-\sum_{\xi=1}^{l} r_{\xi} F\left(c_{\xi}\right)-F\left(\sum_{\zeta=1}^{n} p_{\zeta} a_{\zeta}+\sum_{\eta=1}^{m} q_{\eta} b_{\eta}-\sum_{\xi=1}^{l} r_{\xi} c_{\xi}\right) \\
= & \sum_{\zeta=1}^{n} p_{\zeta} f\left(a_{\zeta}\right)+\sum_{\eta=1}^{m} q_{\eta} f\left(b_{\eta}\right)-\sum_{\xi=1}^{l} r_{\xi} f\left(c_{\xi}\right) d-f\left(\sum_{\zeta=1}^{n} p_{\zeta} a_{\zeta}+\sum_{\eta=1}^{m} q_{\eta} b_{\eta}-\sum_{\xi=1}^{l} r_{\xi} c_{\xi}\right) \\
& -\frac{A}{2}\left\{\sum_{\zeta=1}^{n} p_{\zeta}\left(a_{\zeta}\right)^{2}+\sum_{\eta=1}^{m} q_{\eta}\left(b_{\eta}\right)^{2}-\sum_{\xi=1}^{l} r_{\xi}\left(c_{\xi}\right)^{2}\right\}+\frac{A}{2}\left(\sum_{\zeta=1}^{n} p_{\zeta} a_{\zeta}+\sum_{\eta=1}^{m} q_{\eta} b_{\eta}-\sum_{\xi=1}^{l} r_{\xi} c_{\xi}\right)^{2} .
\end{aligned}
$$

Also, since $f \in K_{c}^{1}(\mathcal{J})$ then there exists a constant $A$ such that $F(x)=f(x)-\frac{A}{2} x^{2}$ is convex on $I \cap[c, \infty)$, hence for $r_{\zeta}, s_{\eta}, t_{\xi} \in I \cap[c, \infty)$ be points such that $t_{\xi} \in \operatorname{conv}\{r, s\}$, so by using inequality (1.1), we have

$$
\begin{aligned}
0 \leqslant & \sum_{\zeta=1}^{n} p_{\zeta} F\left(r_{\zeta}\right)+\sum_{\eta=1}^{m} q_{\eta} F\left(s_{\eta}\right)-\sum_{\xi=1}^{l} r_{\xi} F\left(t_{\xi}\right)-F\left(\sum_{\zeta=1}^{n} p_{\zeta} r_{\zeta}+\sum_{\eta=1}^{m} q_{\eta} s_{\eta}-\sum_{\xi=1}^{l} r_{\xi} t_{\xi}\right) \\
= & \sum_{\zeta=1}^{n} p_{\zeta} f\left(r_{\zeta}\right)+\sum_{\eta=1}^{m} q_{\eta} f\left(s_{\eta}\right)-\sum_{\xi=1}^{l} r_{\xi} f\left(t_{\xi}\right)-f\left(\sum_{\zeta=1}^{n} p_{\zeta} r_{\zeta}+\sum_{\eta=1}^{m} q_{\eta} s_{\eta}-\sum_{\xi=1}^{l} r_{\xi} t_{\xi}\right) \\
& -\frac{A}{2}\left\{\sum_{\zeta=1}^{n} p_{\zeta}\left(r_{\zeta}\right)^{2}+\sum_{\eta=1}^{m} q_{\eta}\left(s_{\eta}\right)^{2}-\sum_{\xi=1}^{l} r_{\xi}\left(t_{\xi}\right)^{2}\right\}+\frac{A}{2}\left(\sum_{\zeta=1}^{n} p_{\zeta} r_{\zeta}+\sum_{\eta=1}^{m} q_{\eta} s_{\eta}-\sum_{\xi=1}^{l} r_{\xi} t_{\xi}\right)^{2} .
\end{aligned}
$$

From above, we have

$$
\begin{aligned}
& \sum_{\zeta=1}^{n} p_{\zeta} f\left(a_{\zeta}\right)+\sum_{\eta=1}^{m} q_{\eta} f\left(b_{\eta}\right)-\sum_{\xi=1}^{l} r_{\xi} f\left(c_{\xi}\right)-f\left(\sum_{\zeta=1}^{n} p_{\zeta} a_{\zeta}+\sum_{\eta=1}^{m} q_{\eta} b_{\eta}-\sum_{\xi=1}^{l} r_{\xi} c_{\xi}\right) \\
& \quad-\frac{A}{2}\left\{\sum_{\zeta=1}^{n} p_{\zeta}\left(a_{\zeta}\right)^{2}+\sum_{\eta=1}^{m} q_{\eta}\left(b_{\eta}\right)^{2}-\sum_{\xi=1}^{l} r_{\xi}\left(c_{\xi}\right)^{2}\right\}+\frac{A}{2}\left(\sum_{\zeta=1}^{n} p_{\zeta} a_{\zeta}+\sum_{\eta=1}^{m} q_{\eta} b_{\eta}-\sum_{\xi=1}^{l} r_{\xi} c_{\xi}\right)^{2} \\
& \leqslant 0 \\
& \leqslant \sum_{\zeta=1}^{n} p_{\zeta} f\left(r_{\zeta}\right)+\sum_{\eta=1}^{m} q_{\eta} f\left(s_{\eta}\right)-\sum_{\xi=1}^{l} r_{\xi} f\left(t_{\xi}\right)-f\left(\sum_{\zeta=1}^{n} p_{\zeta} r_{\zeta}+\sum_{\eta=1}^{m} q_{\eta} s_{\eta}-\sum_{\xi=1}^{l} r_{\xi} t_{\xi}\right)
\end{aligned}
$$




$$
-\frac{A}{2}\left\{\sum_{\zeta=1}^{n} p_{\zeta}\left(r_{\zeta}\right)^{2}+\sum_{\eta=1}^{m} q_{\eta}\left(s_{\eta}\right)^{2}-\sum_{\xi=1}^{l} r_{\xi}\left(t_{\xi}\right)^{2}\right\}+\frac{A}{2}\left(\sum_{\zeta=1}^{n} p_{\zeta} r_{\zeta}+\sum_{\eta=1}^{m} q_{\eta} s_{\eta}-\sum_{\xi=1}^{l} r_{\xi} t_{\xi}\right)^{2} .
$$

So

$$
\begin{aligned}
\sum_{\zeta=1}^{n} p_{\zeta} f\left(a_{\zeta}\right)+\sum_{\eta=1}^{m} q_{\eta} f\left(b_{\eta}\right)-\sum_{\xi=1}^{l} r_{\xi} f\left(c_{\xi}\right)-f\left(\sum_{\zeta=1}^{n} p_{\zeta} a_{\zeta}+\sum_{\eta=1}^{m} q_{\eta} b_{\eta}-\sum_{\xi=1}^{l} r_{\xi} c_{\xi}\right) \\
\quad-\frac{A}{2}\left\{\sum_{\zeta=1}^{n} p_{\zeta}\left(a_{\zeta}\right)^{2}+\sum_{\eta=1}^{m} q_{\eta}\left(b_{\eta}\right)^{2}-\sum_{\xi=1}^{l} r_{\xi}\left(c_{\xi}\right)^{2}\right\}+\frac{A}{2}\left(\sum_{\zeta=1}^{n} p_{\zeta} a_{\zeta}+\sum_{\eta=1}^{m} q_{\eta} b_{\eta}-\sum_{\xi=1}^{l} r_{\xi} c_{\xi}\right)^{2} \\
\leqslant \sum_{\zeta=1}^{n} p_{\zeta} f\left(r_{\zeta}\right)+\sum_{\eta=1}^{m} q_{\eta} f\left(s_{\eta}\right)-\sum_{\xi=1}^{l} r_{\xi} f\left(t_{\xi}\right)-f\left(\sum_{\zeta=1}^{n} p_{\zeta} r_{\zeta}+\sum_{\eta=1}^{m} q_{\eta} s_{\eta}-\sum_{\xi=1}^{l} r_{\xi} t_{\xi}\right) \\
\quad-\frac{A}{2}\left\{\sum_{\zeta=1}^{n} p_{\zeta}\left(r_{\zeta}\right)^{2}+\sum_{\eta=1}^{m} q_{\eta}\left(s_{\eta}\right)^{2}-\sum_{\xi=1}^{l} r_{\xi}\left(t_{\xi}\right)^{2}\right\}+\frac{A}{2}\left(\sum_{\zeta=1}^{n} p_{\zeta} r_{\zeta}+\sum_{\eta=1}^{m} q_{\eta} s_{\eta}-\sum_{\xi=1}^{l} r_{\xi} t_{\xi}\right)^{2}
\end{aligned}
$$

by using (2.1), we get (2.2).

Remark 2.2. From the proof of Theorem 2.1, we have

$$
\begin{aligned}
& \sum_{\zeta=1}^{n} p_{\zeta} f\left(a_{\zeta}\right)+\sum_{\eta=1}^{m} q_{\eta} f\left(b_{\eta}\right)-\sum_{\xi=1}^{l} r_{\xi} f\left(c_{\xi}\right)-f\left(\sum_{\zeta=1}^{n} p_{\zeta} a_{\zeta}+\sum_{\eta=1}^{m} q_{\eta} b_{\eta}-\sum_{\xi=1}^{l} r_{\xi} c_{\xi}\right) \\
& \quad \leqslant \frac{A}{2}\left\{\sum_{\zeta=1}^{n} p_{\zeta}\left(a_{\zeta}\right)^{2}+\sum_{\eta=1}^{m} q_{\eta}\left(b_{\eta}\right)^{2}-\sum_{\xi=1}^{l} r_{\xi}\left(c_{\xi}\right)^{2}\right\}-\frac{A}{2}\left\{\left(\sum_{\zeta=1}^{n} p_{\zeta} a_{\zeta}+\sum_{\eta=1}^{m} q_{\eta} b_{\eta}-\sum_{\xi=1}^{l} r_{\xi} c_{\xi}\right)^{2}\right\}
\end{aligned}
$$

and

$$
\begin{aligned}
& \sum_{\zeta=1}^{n} p_{\zeta} f\left(r_{\zeta}\right)+\sum_{\eta=1}^{m} q_{\eta} f\left(s_{\eta}\right)-\sum_{\xi=1}^{l} r_{\xi} f\left(t_{\xi}\right)-f\left(\sum_{\zeta=1}^{n} p_{\zeta} r_{\zeta}+\sum_{\eta=1}^{m} q_{\eta} s_{\eta}-\sum_{\xi=1}^{l} r_{\xi} t_{\xi}\right) \\
& \geqslant \frac{A}{2}\left\{\sum_{\zeta=1}^{n} p_{\zeta}\left(r_{\zeta}\right)^{2}+\sum_{\eta=1}^{m} q_{\eta}\left(s_{\eta}\right)^{2}-\sum_{\xi=1}^{l} r_{\xi}\left(t_{\xi}\right)^{2}\right\}-\frac{A}{2}\left\{\left(\sum_{\zeta=1}^{n} p_{\zeta} r_{\zeta}+\sum_{\eta=1}^{m} q_{\eta} s_{\eta}-\sum_{\xi=1}^{l} r_{\xi} t_{\xi}\right)^{2}\right\} .
\end{aligned}
$$

So under assumption (2.1), we can get an improvement of (2.2) as follows

$$
\begin{aligned}
& \sum_{\zeta=1}^{n} p_{\zeta} f\left(a_{\zeta}\right)+\sum_{\eta=1}^{m} q_{\eta} f\left(b_{\eta}\right)-\sum_{\xi=1}^{l} r_{\xi} f\left(c_{\xi}\right)-f\left(\sum_{\zeta=1}^{n} p_{\zeta} a_{\zeta}+\sum_{\eta=1}^{m} q_{\eta} b_{\eta}-\sum_{\xi=1}^{l} r_{\xi} c_{\xi}\right) \\
& \leqslant \frac{A}{2}\left\{\sum_{\zeta=1}^{n} p_{\zeta}\left(a_{\zeta}\right)^{2}+\sum_{\eta=1}^{m} q_{\eta}\left(b_{\eta}\right)^{2}-\sum_{\xi=1}^{l} r_{\xi}\left(c_{\xi}\right)^{2}\right\}-\frac{A}{2}\left(\sum_{\zeta=1}^{n} p_{\zeta} a_{\zeta}+\sum_{\eta=1}^{m} q_{\eta} b_{\eta}-\sum_{\xi=1}^{l} r_{\xi} c_{\xi}\right)^{2} \\
& \quad=\frac{A}{2}\left\{\sum_{\zeta=1}^{n} p_{\zeta}\left(r_{\zeta}\right)^{2}+\sum_{\eta=1}^{m} q_{\eta}\left(s_{\eta}\right)^{2}-\sum_{\xi=1}^{l} r_{\xi}\left(t_{\xi}\right)^{2}\right\}-\frac{A}{2}\left(\sum_{\zeta=1}^{n} p_{\zeta} r_{\zeta}+\sum_{\eta=1}^{m} q_{\eta} s_{\eta}-\sum_{\xi=1}^{l} r_{\xi} t_{\xi}\right)^{2} \\
& \leqslant \sum_{\zeta=1}^{n} p_{\zeta} f\left(r_{\zeta}\right)+\sum_{\eta=1}^{m} q_{\eta} f\left(s_{\eta}\right)-\sum_{\xi=1}^{l} r_{\xi} f\left(t_{\xi}\right)-f\left(\sum_{\zeta=1}^{n} p_{\zeta} r_{\zeta}+\sum_{\eta=1}^{m} q_{\eta} s_{\eta}-\sum_{\xi=1}^{l} r_{\xi} t_{\xi}\right) .
\end{aligned}
$$


Assume that $\tilde{\mathrm{a}}=\max _{\zeta}\left\{\boldsymbol{a}_{\zeta}\right\}, \tilde{\mathrm{b}}=\max _{\eta}\left\{\mathrm{b}_{\eta}\right\}, \tilde{\mathrm{c}}=\max _{\xi}\left\{c_{\xi}\right\}$ and $\tilde{\mathrm{r}}=\min _{\zeta}\left\{\mathrm{r}_{\zeta}\right\}, \tilde{\mathrm{s}}=\min _{\eta}\left\{\mathrm{s}_{\eta}\right\}, \tilde{\mathrm{t}}=\min _{\xi}\left\{\mathrm{t}_{\xi}\right\}$. Also, let $\tilde{\tilde{a}}=\max \{\tilde{a}, \tilde{b}, \tilde{\mathrm{c}}\}$ and $\tilde{\tilde{r}}=\min \{\tilde{r}, \tilde{s}, \tilde{\mathrm{t}}\}$. Now, we give the next result which weakens the assumption (2.1) such that inequality (2.4) holds again.

Theorem 2.3. Let $\mathrm{p}_{\zeta}, \mathrm{q}_{\eta}, \mathrm{r}_{\xi} \geqslant 0$ and $\lambda_{\zeta}, \mu_{\eta}, v_{\xi} \geqslant 0$ be coefficients such that their sum $\mathrm{p}=\sum_{\zeta=1}^{n} \mathrm{p}_{\zeta}, \mathrm{q}=$ $\sum_{\eta=1}^{m} q_{\eta}, r=\sum_{\xi=1}^{l} r_{\xi}$ satisfy $p+q-r=1$ and $p, q \in(0,1] ; \lambda=\sum_{\zeta=1}^{n} \lambda_{\zeta}, \mu=\sum_{\eta=1}^{m} \mu_{\eta}, v=\sum_{\xi=1}^{l} v_{\xi}$ satisfy $\lambda+\mu-v=1$ and $\lambda, \mu \in(0,1]$. Let $a_{\zeta}, b_{\eta}, c_{\xi} \in I \subseteq \mathbb{R}$ be points such that $c_{\xi} \in \operatorname{conv}\{a, b\}$ and $r_{\zeta}, s_{\eta}, t_{\xi} \in I \subseteq \mathbb{R}$ be points such that $t_{\xi} \in \operatorname{conv}\{r, s\}$, where

$$
a=\frac{1}{p} \sum_{\zeta=1}^{n} p_{\zeta} a_{\zeta}, b=\frac{1}{q} \sum_{\eta=1}^{m} q_{\eta} b_{\eta}, r=\frac{1}{p} \sum_{\zeta=1}^{n} \lambda_{\zeta} r_{\zeta}, s=\frac{1}{\mu} \sum_{\eta=1}^{m} \mu_{\eta} s_{\eta} .
$$

such that

$$
\tilde{\tilde{a}} \leqslant \tilde{r}
$$

and $\mathrm{f} \in \mathrm{K}_{1}^{\mathrm{c}}(\mathcal{J})$ for some $\mathrm{c} \in[\tilde{\tilde{\mathrm{a}}}, \tilde{\tilde{\mathrm{r}}}]$. Then if

(a)

$$
f_{-}^{\prime \prime}(\tilde{\tilde{a}}) \geqslant 0
$$

and

$$
\begin{aligned}
& \sum_{\zeta=1}^{n} p_{\zeta}\left(a_{\zeta}\right)^{2}+\sum_{\eta=1}^{m} q_{\eta}\left(b_{\eta}\right)^{2}-\sum_{\xi=1}^{l} r_{\xi}\left(c_{\xi}\right)^{2}-\left(\sum_{\zeta=1}^{n} p_{\zeta} a_{\zeta}+\sum_{\eta=1}^{m} q_{\eta} b_{\eta}-\sum_{\xi=1}^{l} r_{\xi} c_{\xi}\right)^{2} \\
& \leqslant \sum_{\zeta=1}^{n} \lambda_{\zeta}\left(r_{\zeta}\right)^{2}+\sum_{\eta=1}^{m} \mu_{\eta}\left(s_{\eta}\right)^{2}-\sum_{\xi=1}^{l} v_{\xi}\left(t_{\xi}\right)^{2}-\left(\sum_{\zeta=1}^{n} \lambda_{\zeta} r_{\zeta}+\sum_{\eta=1}^{m} \mu_{\eta} s_{\eta}-\sum_{\xi=1}^{l} v_{\xi} t_{\xi}\right)^{2}
\end{aligned}
$$

or

(b)

$$
f_{+}^{\prime \prime}(\tilde{\tilde{r}}) \leqslant 0
$$

and

$$
\begin{aligned}
& \sum_{\zeta=1}^{n} p_{\zeta}\left(a_{\zeta}\right)^{2}+\sum_{\eta=1}^{m} q_{\eta}\left(b_{\eta}\right)^{2}-\sum_{\xi=1}^{l} r_{\xi}\left(c_{\xi}\right)^{2}-\left(\sum_{\zeta=1}^{n} p_{\zeta} a_{\zeta}+\sum_{\eta=1}^{m} q_{\eta} b_{\eta}-\sum_{\xi=1}^{l} r_{\xi} c_{\xi}\right)^{2} \\
& \geqslant \sum_{\zeta=1}^{n} \lambda_{\zeta}\left(r_{\zeta}\right)^{2}+\sum_{\eta=1}^{m} \mu_{\eta}\left(s_{\eta}\right)^{2}-\sum_{\xi=1}^{l} v_{\xi}\left(t_{\xi}\right)^{2}-\left(\sum_{\zeta=1}^{n} \lambda_{\zeta} r_{\zeta}+\sum_{\eta=1}^{m} \mu_{\eta} s_{\eta}-\sum_{\xi=1}^{l} v_{\xi} t_{\xi}\right)^{2}
\end{aligned}
$$

or

(c)

$$
\mathrm{f}_{-}^{\prime \prime}(\tilde{\tilde{\mathbf{a}}})<0<\mathrm{f}_{+}^{\prime \prime}(\tilde{\tilde{\mathrm{r}}}) \text { and } \mathrm{f} \text { is 3-convex, }
$$

then (2.2) holds.

Proof. The idea of proof is similar to proof of Theorem 2.1. Hence, by proceeding as in the proof of Theorem 2.1, from the inequality (2.3), we have

$$
\frac{A}{2}\left[\sum_{\zeta=1}^{n} p_{\zeta}\left(r_{\zeta}\right)^{2}+\sum_{\eta=1}^{m} q_{\eta}\left(s_{\eta}\right)^{2}-\sum_{\xi=1}^{l} r_{\xi}\left(t_{\xi}\right)^{2}\right]-\frac{A}{2}\left(\sum_{\zeta=1}^{n} p_{\zeta} r_{\zeta}+\sum_{\eta=1}^{m} q_{\eta} s_{\eta}-\sum_{\xi=1}^{l} r_{\xi} t_{\xi}\right)^{2}
$$




$$
\begin{aligned}
& -\frac{A}{2}\left[\sum_{\zeta=1}^{n} p_{\zeta}\left(a_{\zeta}\right)^{2}+\sum_{\eta=1}^{m} q_{\eta}\left(b_{\eta}\right)^{2}-\sum_{\xi=1}^{l} r_{\xi}\left(c_{\xi}\right)^{2}\right]+\frac{A}{2}\left(\sum_{\zeta=1}^{n} p_{\zeta} a_{\zeta}+\sum_{\eta=1}^{m} q_{\eta} b_{\eta}-\sum_{\xi=1}^{l} r_{\xi} c_{\xi}\right)^{2} \\
\leqslant & \sum_{\zeta=1}^{n} p_{\zeta} f\left(r_{\zeta}\right)+\sum_{\eta=1}^{m} q_{\eta} f\left(s_{\eta}\right)-\sum_{\xi=1}^{l} r_{\xi} f\left(t_{\xi}\right)-f\left(\sum_{\zeta=1}^{n} p_{\zeta} r_{\zeta}+\sum_{\eta=1}^{m} q_{\eta} s_{\eta}-\sum_{\xi=1}^{l} r_{\xi} t_{\xi}\right) \\
& -\left\{\sum_{\zeta=1}^{n} p_{\zeta} f\left(a_{\zeta}\right)+\sum_{\eta=1}^{m} q_{\eta} f\left(b_{\eta}\right)-\sum_{\xi=1}^{l} r_{\xi} f\left(c_{\xi}\right)\right\}+f\left(\sum_{\zeta=1}^{n} p_{\zeta} a_{\zeta}+\sum_{\eta=1}^{m} q_{\eta} b_{\eta}-\sum_{\xi=1}^{l} r_{\xi} c_{\xi}\right) .
\end{aligned}
$$

Now, due to the concavity of $F$ on $I \cap(-\infty, c]$ and convexity of $F$ on $I \cap[c, \infty)$, so for every distinct points $x_{\eta} \in[a, \tilde{\tilde{a}}]$ and $y_{\eta} \in[\tilde{r}, b], j=1,2,3$, we have

$$
\left[x_{1}, x_{2}, x_{3}\right] f \leqslant A \leqslant\left[y_{1}, y_{2}, y_{3}\right] f .
$$

Letting $x_{\eta} \nearrow \tilde{\tilde{a}}$ and $y_{\eta} \searrow \tilde{\tilde{r}}$, we get (if exists)

$$
f_{-}^{\prime \prime}(\tilde{\tilde{a}}) \leqslant A \leqslant f_{+}^{\prime \prime}(\tilde{\tilde{r}}) .
$$

Therefore, if assumptions (a) or (b) holds, then

$$
\begin{aligned}
& \frac{A}{2}\left[\sum_{\zeta=1}^{n} p_{\zeta}\left(r_{\zeta}\right)^{2}+\sum_{\eta=1}^{m} q_{\eta}\left(s_{\eta}\right)^{2}-\sum_{\xi=1}^{l} r_{\xi}\left(t_{\xi}\right)^{2}\right]-\frac{A}{2}\left(\sum_{\zeta=1}^{n} p_{\zeta} r_{\zeta}+\sum_{\eta=1}^{m} q_{\eta} s_{\eta}-\sum_{\xi=1}^{l} r_{\xi} t_{\xi}\right)^{2} \\
& \quad-\frac{A}{2}\left\{\sum_{\zeta=1}^{n} p_{\zeta}\left(a_{\zeta}\right)^{2}+\sum_{\eta=1}^{m} q_{\eta}\left(b_{\eta}\right)^{2}-\sum_{\xi=1}^{l} r_{\xi}\left(c_{\xi}\right)^{2}\right\}+\frac{A}{2}\left(\sum_{\zeta=1}^{n} p_{\zeta} a_{\zeta}+\sum_{\eta=1}^{m} q_{\eta} b_{\eta}-\sum_{\xi=1}^{l} r_{\xi} c_{\xi}\right)^{2}
\end{aligned}
$$

is positive and we conclude the result. If the assumption (c) holds, the $f_{-}^{\prime \prime}$ is left continuous, $f_{+}^{\prime \prime}$ is right continuous, they are both non-decreasing and $f_{-}^{\prime \prime} \leqslant f_{+}^{\prime \prime}$. Therefore, there exists $\tilde{c} \in[\tilde{\tilde{a}}, \tilde{\tilde{r}}]$ such that $f \in K_{1}^{\tilde{c}}(I)$ with associated constant $\tilde{A}=0$ and again, we can deduce the result.

Remark 2.4. Again from the proof of Theorem 2.1, we obtain the inequalities (2.4) and (2.5). Now, under assumption (a), (b), or (c) of Theorem 2.3, A is positive or negative or zero, respectively due to argument discussed in the proof. Therefore, we get a better improvement of (2.2) then (2.6) in this case as follows

$$
\begin{aligned}
& \sum_{\zeta=1}^{n} p_{\zeta} f\left(a_{\zeta}\right)+\sum_{\eta=1}^{m} q_{\eta} f\left(b_{\eta}\right)-\sum_{\xi=1}^{l} r_{\xi} f\left(c_{\xi}\right)-f\left(\sum_{\zeta=1}^{n} p_{\zeta} a_{\zeta}+\sum_{\eta=1}^{m} q_{\eta} b_{\eta}-\sum_{\xi=1}^{l} r_{\xi} c_{\xi}\right) \\
& \leqslant \frac{A}{2}\left\{\sum_{\zeta=1}^{n} p_{\zeta}\left(a_{\zeta}\right)^{2}+\sum_{\eta=1}^{m} q_{\eta}\left(b_{\eta}\right)^{2}-\sum_{\xi=1}^{l} r_{\xi}\left(c_{\xi}\right)^{2}\right\}-\frac{A}{2}\left(\sum_{\zeta=1}^{n} p_{\zeta} a_{\zeta}+\sum_{\eta=1}^{m} q_{\eta} b_{\eta}-\sum_{\xi=1}^{l} r_{\xi} c_{\xi}\right)^{2} \\
& \leqslant \frac{A}{2}\left\{\sum_{\zeta=1}^{n} p_{\zeta}\left(r_{\zeta}\right)^{2}+\sum_{\eta=1}^{m} q_{\eta}\left(s_{\eta}\right)^{2}-\sum_{\xi=1}^{l} r_{\xi}\left(t_{\xi}\right)^{2}\right\}-\frac{A}{2}\left(\sum_{\zeta=1}^{n} p_{\zeta} r_{\zeta}+\sum_{\eta=1}^{m} q_{\eta} s_{\eta}-\sum_{\xi=1}^{l} r_{\xi} t_{\xi}\right)^{2} \\
& \leqslant \sum_{\zeta=1}^{n} p_{\zeta} f\left(r_{\zeta}\right)+\sum_{\eta=1}^{m} q_{\eta} f\left(s_{\eta}\right)-\sum_{\xi=1}^{l} r_{\xi} f\left(t_{\xi}\right)-f\left(\sum_{\zeta=1}^{n} p_{\zeta} r_{\zeta}+\sum_{\eta=1}^{m} q_{\eta} s_{\eta}-\sum_{\xi=1}^{l} r_{\xi} t_{\xi}\right) .
\end{aligned}
$$

Under the assumption of Theorem 2.1 with $f \in K_{2}^{c}(\mathcal{J})$, the reverse of inequality (2.2) holds. Now, we give only the statement by weakening assumptions of Theorem 2.1 under which the reverse of inequality (2.2) holds for $f \in K_{2}^{c}(I)$. 
Theorem 2.5. Let $\mathrm{p}_{\zeta}, \mathrm{q}_{\eta}, \mathrm{r}_{\xi} \geqslant 0$ and $\lambda_{\zeta}, \mu_{\eta}, v_{\xi} \geqslant 0$ be coefficients such that their sum $\mathrm{p}=\sum_{\zeta=1}^{n} \mathrm{p}_{\zeta}, \mathrm{q}=$ $\sum_{\eta=1}^{m} q_{\eta}, r=\sum_{\xi=1}^{l} r_{\xi}$ satisfy $p+q-r=1$ and $p, q \in(0,1] ; \lambda=\sum_{\zeta=1}^{n} \lambda_{\zeta}, \mu=\sum_{\eta=1}^{m} \mu_{\eta}, v=\sum_{\xi=1}^{l} v_{\xi}$ satisfy $\lambda+\mu-v=1$ and $\lambda, \mu \in(0,1]$. Let $a_{\zeta}, b_{\eta}, c_{\xi} \in I \subseteq \mathbb{R}$ be points such that $c_{\xi} \in \operatorname{conv}\{a, b\}$ and $\mathrm{r}_{\zeta}, \mathrm{s}_{\eta}, \mathrm{t}_{\xi} \in \mathrm{I} \subseteq \mathbb{R}$ be points such that $\mathrm{t}_{\xi} \in \operatorname{conv}\{\mathrm{r}, \mathrm{s}\}$, where

$$
a=\frac{1}{p} \sum_{\zeta=1}^{n} p_{\zeta} a_{\zeta}, b=\frac{1}{q} \sum_{\eta=1}^{m} q_{\eta} b_{\eta}, r=\frac{1}{p} \sum_{\zeta=1}^{n} \lambda_{\zeta} r_{\zeta}, s=\frac{1}{\mu} \sum_{\eta=1}^{m} \mu_{\eta} s_{\eta},
$$

such that

$$
\tilde{\tilde{a}} \leqslant \tilde{\tilde{r}}
$$

and $\mathrm{f} \in \mathrm{K}_{2}^{\mathrm{c}}(\mathcal{J})$ for some $\mathrm{c} \in[\tilde{\tilde{\mathrm{a}}}, \tilde{\tilde{\mathrm{r}}}]$. Then if

(a)

$$
f_{-}^{\prime \prime}(\tilde{\tilde{a}}) \leqslant 0
$$

and

$$
\begin{aligned}
& \sum_{\zeta=1}^{n} p_{\zeta}\left(a_{\zeta}\right)^{2}+\sum_{\eta=1}^{m} q_{\eta}\left(b_{\eta}\right)^{2}-\sum_{\xi=1}^{l} r_{\xi}\left(c_{\xi}\right)^{2}-\left(\sum_{\zeta=1}^{n} p_{\zeta} a_{\zeta}+\sum_{\eta=1}^{m} q_{\eta} b_{\eta}-\sum_{\xi=1}^{l} r_{\xi} c_{\xi}\right)^{2} \\
& \geqslant \sum_{\zeta=1}^{n} \lambda_{\zeta}\left(r_{\zeta}\right)^{2}+\sum_{\eta=1}^{m} \mu_{\eta}\left(s_{\eta}\right)^{2}-\sum_{\xi=1}^{l} v_{\xi}\left(t_{\xi}\right)^{2}-\left(\sum_{\zeta=1}^{n} \lambda_{\zeta} r_{\zeta}+\sum_{\eta=1}^{m} \mu_{\eta} s_{\eta}-\sum_{\xi=1}^{l} v_{\xi} t_{\xi}\right)^{2},
\end{aligned}
$$

or

(b)

$$
f_{+}^{\prime \prime}(\tilde{\tilde{r}}) \geqslant 0
$$

and

$$
\begin{aligned}
& \sum_{\zeta=1}^{n} p_{\zeta}\left(a_{\zeta}\right)^{2}+\sum_{\eta=1}^{m} q_{\eta}\left(b_{\eta}\right)^{2}-\sum_{\xi=1}^{l} r_{\xi}\left(c_{\xi}\right)^{2}-\left(\sum_{\zeta=1}^{n} p_{\zeta} a_{\zeta}+\sum_{\eta=1}^{m} q_{\eta} b_{\eta}-\sum_{\xi=1}^{l} r_{\xi} c_{\xi}\right)^{2} \\
& \leqslant \sum_{\zeta=1}^{n} \lambda_{\zeta}\left(r_{\zeta}\right)^{2}+\sum_{\eta=1}^{m} \mu_{\eta}\left(s_{\eta}\right)^{2}-\sum_{\xi=1}^{l} v_{\xi}\left(t_{\xi}\right)^{2}-\left(\sum_{\zeta=1}^{n} \lambda_{\zeta} r_{\zeta}+\sum_{\eta=1}^{m} \mu_{\eta} s_{\eta}-\sum_{\xi=1}^{l} v_{\xi} t_{\xi}\right)^{2},
\end{aligned}
$$

or

(c)

$$
f_{-}^{\prime \prime}(\tilde{\tilde{a}})<0<f_{+}^{\prime \prime}(\tilde{\tilde{r}}) \text { and } f \text { is 3-concave, }
$$

then reverse of (2.2) holds.

Remark 2.6. From the proof of the Theorem 2.5, we obtain the reverse of inequalities (2.4) and (2.5). Now, due to the convexity of $F$ on $I \cap(-\infty, c]$ and concavity of $F$ on $I \cap[c, \infty)$, for every distinct points $x_{\eta} \in[a, \tilde{a}]$ and $y_{\eta} \in[\tilde{r}, b], \eta=1,2,3$, we have

$$
\left[x_{1}, x_{2}, x_{3}\right] f \geqslant A \geqslant\left[y_{1}, y_{2}, y_{3}\right] f .
$$

Letting $x_{\eta} \nearrow \tilde{\tilde{a}}$ and $y_{\eta} \searrow \tilde{\tilde{r}}$, we get (if exists)

$$
f_{-}^{\prime \prime}(\tilde{\tilde{a}}) \geqslant A \geqslant f_{+}^{\prime \prime}(\tilde{\tilde{r}}) .
$$


Now, under assumption (a), (b), or (c) of Theorem 2.3, A is negative or positive or zero, respectively due to argument discussed above. Therefore, we get a better improvement in this case as follows

$$
\begin{aligned}
& \sum_{\zeta=1}^{n} p_{\zeta} f\left(a_{\zeta}\right)+\sum_{\eta=1}^{m} q_{\eta} f\left(b_{\eta}\right)-\sum_{\xi=1}^{l} r_{\xi} f\left(c_{\xi}\right)-f\left(\sum_{\zeta=1}^{n} p_{\zeta} a_{\zeta}+\sum_{\eta=1}^{m} q_{\eta} b_{\eta}-\sum_{\xi=1}^{l} r_{\xi} c_{\xi}\right) \\
& \geqslant \frac{A}{2}\left\{\sum_{\zeta=1}^{n} p_{\zeta}\left(a_{\zeta}\right)^{2}+\sum_{\eta=1}^{m} q_{\eta}\left(b_{\eta}\right)^{2}-\sum_{\xi=1}^{l} r_{\xi}\left(c_{\xi}\right)^{2}\right\}-\frac{A}{2}\left\{\left(\sum_{\zeta=1}^{n} p_{\zeta} a_{\zeta}+\sum_{\eta=1}^{m} q_{\eta} b_{\eta}-\sum_{\xi=1}^{l} r_{\xi} c_{\xi}\right)^{2}\right\} \\
& \geqslant \frac{A}{2}\left\{\sum_{\zeta=1}^{n} p_{\zeta}\left(r_{\zeta}\right)^{2}+\sum_{\eta=1}^{m} q_{\eta}\left(s_{\eta}\right)^{2}-\sum_{\xi=1}^{l} r_{\xi}\left(t_{\xi}\right)^{2}\right\}-\frac{A}{2}\left\{\left(\sum_{\zeta=1}^{n} p_{\zeta} r_{\zeta}+\sum_{\eta=1}^{m} q_{\eta} s_{\eta}-\sum_{\xi=1}^{l} r_{\xi} t_{\xi}\right)^{2}\right\} \\
& \geqslant \sum_{\zeta=1}^{n} p_{\zeta} f\left(r_{\zeta}\right)+\sum_{\eta=1}^{m} q_{\eta} f\left(s_{\eta}\right)-\sum_{\xi=1}^{l} r_{\xi} f\left(t_{\xi}\right)-f\left(\sum_{\zeta=1}^{n} p_{\zeta} r_{\zeta}+\sum_{\eta=1}^{m} q_{\eta} s_{\eta}-\sum_{\xi=1}^{l} r_{\xi} t_{\xi}\right) .
\end{aligned}
$$

Theorem 2.7. Let $\mathcal{J} \subseteq \mathbb{R}$ be a closed interval, let $[\mathrm{a}, \mathrm{b}] \subseteq \mathcal{J}$, let function $\mathrm{g}_{\zeta} \in \mathbb{X}_{[\mathrm{a}, \mathrm{b}]}$ and function $\mathrm{h}_{\zeta} \in \mathbb{X}_{\mathcal{J} \backslash(\mathrm{a}, \mathrm{b})}$ for $\zeta=1$, 2. Let $\mathrm{f} \in \mathrm{K}_{1}^{\mathrm{c}}(\mathcal{J})$ be continuous function such that $\mathrm{f}\left(\mathrm{g}_{\zeta}\right), \mathrm{f}\left(\mathrm{h}_{\zeta}\right) \in \mathbb{X}$. If a pair of unital positive linear functionals $\mathrm{L}, \mathrm{H}: \mathbb{X} \rightarrow \mathbb{R}$ satisfies

$$
\mathrm{L}\left(\mathrm{g}_{\zeta}\right)=\mathrm{H}\left(\mathrm{h}_{\zeta}\right) \text { and } \mathrm{H}\left(\mathrm{h}_{1}^{2}\right)-\mathrm{L}\left(\mathrm{g}_{1}^{2}\right)=\mathrm{H}\left(\mathrm{h}_{2}^{2}\right)-\mathrm{L}\left(\mathrm{g}_{2}^{2}\right), \quad \zeta=1,2,
$$

then inequality

$$
H\left(f\left(h_{1}\right)\right)-L\left(f\left(g_{1}\right)\right) \leqslant H\left(f\left(h_{2}\right)\right)-L\left(f\left(g_{2}\right)\right)
$$

holds.

Proof. Since $f \in K_{1}^{c}(\mathcal{J})$, there exists a constant $A$ such that $F(x)=f(x)-\frac{A}{2} x^{2}$ is concave on $\mathcal{J} \cap(-\infty, c]$, therefore by reverse of (1.3) for $F$ on $\mathcal{J} \cap(-\infty, c]$, we get

$$
0 \geqslant H\left(F\left(h_{1}\right)\right)-L\left(F\left(g_{1}\right)\right)=H\left(f\left(h_{1}\right)\right)-L\left(f\left(g_{1}\right)\right)-\frac{A}{2}\left(H\left(h_{1}^{2}\right)-L\left(g_{1}^{2}\right)\right) .
$$

Also, since $F(x)=f(x)-\frac{A}{2} x^{2}$ is convex on $\mathcal{J} \cap[c, \infty)$, therefore by (1.3) for $F$ on $\mathcal{J} \cap(-\infty, c]$, we get

$$
0 \leqslant H\left(F\left(h_{2}\right)\right)-L\left(F\left(g_{2}\right)\right)=H\left(f\left(h_{2}\right)\right)-L\left(f\left(g_{2}\right)\right)-\frac{A}{2}\left(H\left(h_{2}^{2}\right)-L\left(g_{2}^{2}\right)\right) .
$$

From above, we have

$$
H\left(f\left(h_{1}\right)\right)-L\left(f\left(g_{1}\right)\right)-\frac{A}{2}\left(H\left(h_{1}^{2}\right)-L\left(g_{1}^{2}\right)\right) \leqslant 0 \leqslant H\left(f\left(h_{2}\right)\right)-L\left(f\left(g_{2}\right)\right)-\frac{A}{2}\left(H\left(h_{2}^{2}\right)-L\left(g_{2}^{2}\right)\right) .
$$

So

$$
H\left(f\left(h_{1}\right)\right)-L\left(f\left(g_{1}\right)\right)-\frac{A}{2}\left(H\left(h_{1}^{2}\right)-L\left(g_{1}^{2}\right)\right) \leqslant H\left(f\left(h_{2}\right)\right)-L\left(f\left(g_{2}\right)\right)-\frac{A}{2}\left(H\left(h_{2}^{2}\right)-L\left(g_{2}^{2}\right)\right),
$$

therefore, by the use of (2.7), we get (2.8).

Remark 2.8. From the proof of the Theorem 2.7, we have

$$
H\left(f\left(h_{1}\right)\right)-L\left(f\left(g_{1}\right)\right) \leqslant \frac{A}{2}\left(H\left(h_{1}^{2}\right)-L\left(g_{1}^{2}\right)\right)
$$

and

$$
H\left(f\left(h_{2}\right)\right)-L\left(f\left(g_{2}\right)\right) \geqslant \frac{A}{2}\left(H\left(h_{2}^{2}\right)-L\left(g_{2}^{2}\right)\right) .
$$

So, under assumption (2.7), we can get a better improvement of (2.8) as follow

$$
H\left(f\left(h_{1}\right)\right)-L\left(f\left(g_{1}\right)\right) \leqslant \frac{A}{2}\left(H\left(h_{1}^{2}\right)-L\left(g_{1}^{2}\right)\right)\left(=\frac{A}{2}\left(H\left(h_{2}^{2}\right)-L\left(g_{2}^{2}\right)\right)\right) \leqslant H\left(f\left(h_{2}\right)\right)-L\left(f\left(g_{2}\right)\right) .
$$


Corollary 2.9. Let $\mathcal{J} \subseteq \mathbb{R}$ be a closed interval, let $[\mathrm{a}, \mathrm{b}] \subseteq \mathcal{J}$, let function $\mathrm{g}_{\zeta} \in \mathbb{X}_{[\mathrm{a}, \mathrm{b}]}$ for $\zeta=1$, 2. Let $\mathrm{f} \in \mathrm{K}_{1}^{\mathrm{c}}(\mathrm{J})$ be continuous function such that $f\left(g_{\zeta}\right) \in \mathbb{X}$. If a unital positive linear functionals $L: \mathbb{X} \rightarrow \mathbb{R}$ satisfies implication (2.7) $\Rightarrow$ (2.8) for $\mathrm{L}=\mathrm{H}$ such that

$$
\mathrm{L}\left(\mathrm{g}_{1}^{2}\right)-\left(\mathrm{L}\left(\mathrm{g}_{1}\right)\right)^{2}=\mathrm{L}\left(\mathrm{g}_{2}^{2}\right)-\left(\mathrm{L}\left(\mathrm{g}_{2}\right)\right)^{2},
$$

then following inequality holds

$$
L\left(f\left(g_{1}\right)\right)-f\left(L\left(g_{1}\right)\right) \leqslant L\left(f\left(g_{2}\right)\right)-f\left(L\left(g_{2}\right)\right) .
$$

Corollary 2.10. Let $\left[a_{1}, b_{1}\right] \subseteq \cdots \subseteq\left[a_{n-1}, b_{n-1}\right] \subseteq \mathcal{J}$ and let functions $g_{1}, h_{1} \in \mathbb{X}_{\left[a_{1}, b_{1}\right]}$, let $g_{\xi}$, $h_{\xi} \in \mathbb{X}_{\left[a_{\xi}, b_{\xi}\right] \backslash\left(a_{\xi-1}, b_{\xi-1}\right)}$ for $\xi=2, \ldots, n-1$, and functions $g_{n}, h_{n} \in \mathbb{X}_{\mathcal{J} \backslash\left(a_{n-1}, b_{n-1}\right)}$. Let $f \in K_{1}^{c}(\mathcal{J})$ be continuous function such that $\mathrm{f}\left(\mathrm{g}_{\zeta}\right), \mathrm{f}\left(\mathrm{h}_{\zeta}\right) \in \mathbb{X}$. If an n-tuple of unital positive linear functionals $\mathrm{L}_{\zeta}: \mathbb{X} \rightarrow \mathbb{R}$ satisfies

$$
\mathrm{L}_{\zeta}\left(\mathrm{g}_{\zeta}\right)=\mathrm{L}_{\zeta+1}\left(\mathrm{~g}_{\zeta+1}\right) \text { and } \mathrm{L}_{\zeta}\left(\mathrm{h}_{\zeta}\right)=\mathrm{L}_{\zeta+1}\left(\mathrm{~h}_{\zeta+1}\right) \text { for } \zeta=1, \ldots, \mathrm{n}-1 \text {, }
$$

such that

$$
\mathrm{L}_{\zeta+1}\left(\mathrm{~g}_{\zeta+1}^{2}\right)-\mathrm{L}_{\zeta}\left(\mathrm{g}_{\zeta}^{2}\right)=\mathrm{L}_{\zeta+1}\left(\mathrm{~h}_{\zeta+1}^{2}\right)-\mathrm{L}_{\zeta}\left(\mathrm{h}_{\zeta}^{2}\right)
$$

then

$$
L_{\zeta+1} f\left(g_{\zeta+1}\right)-L_{\zeta} f\left(g_{\zeta}\right) \leqslant L_{\zeta+1} f\left(h_{\zeta+1}\right)-L_{\zeta} f\left(h_{\zeta}\right) \text { for } \zeta=1, \ldots, n-1 .
$$

Corollary 2.11. Let functions $g_{\zeta}, h_{\zeta} \in \mathbb{X}_{\mathcal{J}}$ for $\zeta=1, \ldots, n$. Let $f \in K_{1}^{c}(\mathcal{J})$ be continuous function such that $f\left(g_{\zeta}\right), f\left(h_{\zeta}\right) \in \mathbb{X}$. Then every $n$-tuple of positive linear functionals $L_{\zeta}: \mathbb{X} \rightarrow \mathbb{R}$ with $\sum_{\zeta=1}^{n} L_{\zeta}(1)=1$ such that

$$
\sum_{\zeta=1}^{n} L_{\zeta}\left(\left(g_{\zeta}\right)^{2}\right)-\left(\sum_{\zeta=1}^{n} L_{\zeta}\left(g_{\zeta}\right)\right)^{2}=\sum_{\zeta=1}^{n} L_{\zeta}\left(\left(h_{\zeta}\right)^{2}\right)-\left(\sum_{\zeta=1}^{n} L_{\zeta}\left(h_{\zeta}\right)\right)^{2},
$$

satisfies the inclusion

$$
\sum_{\zeta=1}^{n} L_{\zeta}\left(g_{\zeta}\right), \sum_{\zeta=1}^{n} L_{\zeta}\left(h_{\zeta}\right) \in \mathcal{J}
$$

and the inequality

$$
\sum_{\zeta=1}^{n} L_{\zeta}\left(f\left(g_{\zeta}\right)\right)-f\left(\sum_{\zeta=1}^{n} L_{\zeta}\left(g_{\zeta}\right)\right) \leqslant \sum_{\zeta=1}^{n} L_{\zeta}\left(f\left(h_{\zeta}\right)\right)-f\left(\sum_{\zeta=1}^{n} L_{\zeta}\left(h_{\zeta}\right)\right) .
$$

Theorem 2.12. Let $[\mathrm{a}, \mathrm{b}] \subset \mathcal{J}, \mathrm{g}_{\zeta}, \mathrm{g}_{\zeta}^{*} \in \mathbb{X}_{[\mathrm{a}, \mathrm{b}]}$ for $\zeta=1, \ldots, \mathrm{n}$ and $\mathrm{h}_{\eta}, \mathrm{h}_{\eta}^{*} \in \mathbb{X}_{\mathcal{J} \backslash(\mathrm{a}, \mathrm{b})}$ for $\eta=1, \ldots, \mathrm{m}$. Also, let $\mathrm{f} \in \mathrm{K}_{1}^{\mathrm{c}}(\mathcal{J})$ be continuous function such that $\mathrm{f}\left(\mathrm{g}_{\zeta}\right), \mathrm{f}\left(\mathrm{g}_{\zeta}^{*}\right), \mathrm{f}\left(\mathrm{h}_{\eta}\right), \mathrm{f}\left(\mathrm{h}_{\eta}^{*}\right) \in \mathbb{X}$. If two pair of $\mathrm{n}$-tuple of positive linear functionals $\mathrm{L}_{\zeta}, \mathrm{L}_{\zeta}^{*}, \mathrm{H}_{\eta}, \mathrm{H}_{\eta}^{*}: \mathbb{X} \rightarrow \mathbb{R}$ with $\sum_{\zeta=1}^{\mathfrak{n}} \mathrm{L}_{\zeta}(1)=\sum_{1=1}^{n} \mathrm{~L}_{\zeta}^{*}(1)=\sum_{\eta=1}^{m} \mathrm{H}_{\eta}(1)=\sum_{\eta=1}^{\mathrm{m}} \mathrm{H}_{\eta}^{*}(1)=1$ satisfy

$$
\sum_{\eta=1}^{m} H_{\eta}\left(h_{\eta}\right)=\sum_{\zeta=1}^{n} L_{\zeta}\left(g_{\zeta}\right) \text { and } \sum_{\eta=1}^{m} H_{\eta}^{*}\left(h_{\eta}^{*}\right)=\sum_{\zeta=1}^{n} L_{\zeta}^{*}\left(g_{\zeta}^{*}\right)
$$

and

$$
\sum_{\eta=1}^{m} H_{\eta}\left(\left(h_{\eta}\right)^{2}\right)-\sum_{\zeta=1}^{n} L_{\zeta}\left(\left(g_{\zeta}\right)^{2}\right)=\sum_{\eta=1}^{m} H_{\eta}^{*}\left(\left(h_{\eta}^{*}\right)^{2}\right)-\sum_{\zeta=1}^{n} L_{\zeta}^{*}\left(\left(g_{\zeta}^{*}\right)^{2}\right),
$$

then

$$
\sum_{\eta=1}^{m} H_{\eta} f\left(h_{\eta}\right)-\sum_{\zeta=1}^{n} L_{\zeta} f\left(g_{\zeta}\right) \leqslant \sum_{\eta=1}^{m} H_{\eta}^{*} f\left(h_{\eta}^{*}\right)-\sum_{\zeta=1}^{n} L_{\zeta}^{*} f\left(g_{\zeta}^{*} s\right) .
$$




\section{Conclusion}

In this paper, we presented more generalized extension of Jensen's type inequality to affine combinations and functional form of Jensen's type inequality for non-convex functions. We hope that our results will stimulate other researcher to explore more exciting results in literature.

\section{Data availability}

No data were used to support this study.

\section{Acknowledgment}

Authors would like to thank the editor and anonymous referee for their valuable comments and suggestions. The authors are grateful to the Basque Government by its support through Grant IT1207/19. This research article is partially supported by Higher Education Commission of Pakistan too.

\section{References}

[1] I. A. Baloch, J. Pečarič, M. Praljak, Generalization of Levinson's inequality, J. Math. Inequal., 9 (2015), 571-586. 1, 1

[2] C. P. Niculescu, L.-E. Persson, Convex functions and their applications, Springer-Verlag, New York, (2006).

[3] Z. Pavić, Generalization of the Functional form of Jensen's inequality, J. Adv. Inequal. Appl., 2014 (2014), 12 pages. 1, 1

[4] Z. Pavić, Certain inequalities for convex functions, J. Math. Inequal., 9 (2015), 1349-1364. 1

[5] J. E. Pečarič, F. Proschan, Y. L. Tong, Convex Functions, Partial Orderings and Statistical Applications, Academic Press, Boston, (1991). 